\title{
End-user oriented strategies to facilitate multi-organizational adoption of emergency management information systems
}

\author{
Ignacio Aedo ${ }^{\mathrm{a}}$, Paloma Díaz ${ }^{\mathrm{a}, *}$, John M. Carroll ${ }^{\mathrm{b}}$, Gregorio Convertino ${ }^{\mathrm{c}}$, Mary Beth Rosson ${ }^{\mathrm{b}}$ \\ a Computer Science Department, Universidad Carlos III de Madrid, Avda de la Universidad 30, 28911 Leganés, Spain \\ ${ }^{\mathrm{b}}$ College of Information Science and Technology, Pennsylvania State University, University Park, PA 16802, USA \\ ${ }^{\mathrm{c}}$ Palo Alto Research Center, 3333 Coyote Hill Road, Palo Alto, CA 94304, USA
}

Keywords:

Emergency management information systems

IS acceptance

Participatory design

\begin{abstract}
A B S T R A C T
Response to large scale emergencies is a cooperative process that requires the active and coordinated participation of a variety of functionally independent agencies operating in adjacent regions. In practice, this essential cooperation is sometimes not attained or is reduced due to poor information sharing, non fluent communication flows, and lack of coordination. We report an empirical study of IT mediated cooperation among Spanish response agencies and we describe the challenges of adoption, information sharing, com munication flows, and coordination among agencies that do not share a unity of command. We analyze three strategies aimed at supporting acceptance and surmounting political, organizational and personal distrust or skepticism: participatory design, advanced collab orative tools inducing cognitive absorption, and end user communities of practice.
\end{abstract}

\section{Introduction}

Emergency management is a cooperative process that requires the active and coordinated participation of different kinds of agents, including governmental and non-governmental agencies and corps, communities, volunteers and citizens (Carver \& Turoff, 2007; Waugh \& Streib, 2006). This work activity can be analyzed into the phases of preparedness, response, recovery and mitigation. In this paper, we will focus on the response phase, and more specifically on cooperation among functionally independent governmental agencies operating in adjacent geographical regions. Such agencies are intended to operate as peers; they are organized to have similar functions and competencies. But in many situations these peer agencies are not organized under any single oversight authority; they may have no hierarchical dependencies; they may not be part of a unified command and control coordination structure. Accordingly, each agency can decide whether and how to cooperate.

In large-scale emergencies, high level of mutual independence of this sort can be problematic. Many real world cases have demonstrated that flaws in the cooperation among emergency response agencies can lead to inefficient and ineffective outcomes (García-González, 2007; Waugh \& Streib, 2006). Information technology (IT) solutions have been developed to enhance inter-agency flows of information, communication and coordination (the ICC triad), including NIMS-IRIS in USA (FEMA, 2008a), CECIS for the European Union (European Community, 2008) and ARCE for the Latin American Community (Aedo, Díaz, Fernández, \& de Castro, 2002). However, experience demonstrates that the coordination of independent agencies cannot be achieved with a unique command and control center. For example, the European Union coordinates international cooperation through its MIC center, a policy whose efficacy has been often questioned specially in real emergencies like the wild fires in Greece during 2007 where more than 60 people died and all European contributors were very critic with

\footnotetext{
* Corresponding author.

E mail address: pdp@inf.uc3m.es (P. Díaz).
} 
the utility of that role of central coordinator played by the MIC. In this paper we focus on systems that make it possible to coordinate the action of independent agencies without imposing any artificial unique command and control structure. Our goal is to deploy emergency management information systems (EMIS so forth) that might contribute to support the coordination of loosely coupled tasks among different agencies. Such coordination tasks have fit dependencies in terms of the Coordination Theory (Malone, 2001), that is, they are performed in an independent way and all them are aimed at reaching a specific goal that, in our case, is a more efficient response to an emergency.

EMIS might also support a fluent information flow among agencies to improve awareness, that is, to help independent agencies to understand what's going on in the emergency, what's needed and what are other agencies doing to avoid duplicities. It's worth mentioning there that this is not a typical case of situational awareness since we are not dealing with an individual responder who has to evaluate a number of cues and signals to take an immediate decision; our focus is on operators working on decision centers of governmental agencies who have to understand which are the needs other agency has, what are other agencies doing and what can her agency do to contribute to mitigate the effects of a specific disaster. In this sense, we will be dealing with activity awareness as described in (Carroll, Neale, Isenhour, Rosson, \& McCrickard, 2003) more than with moment-to-moment situational awareness as defined in (Endsley, 2000). It's worth mentioning here that this is not either a collaborative work. Agencies might have different and competing goals and there isn't a unique command and control center, therefore, this is not a case of highly coupled collaboration and group decision-making but of communication and coordination among independent agencies. In any case, EMIS can convey the right information at the right moment to the right person and in a way that is cognitively and physically usable for its end-users (Endsley, 2000), so that operators will have enough resources as to understand the situation (situation assessment) and come up with an adequate mental model of it (situation awareness). However, we shouldn't forget that situation awareness is not created by the system, but it is an status of the operator of the system and, therefore, there are many other factors that will influence the operatoŕs conception of the situation (i.e., her situation awareness) as well as the evaluation of such situation and of the required actions (i.e., her situation assessment), including previous experience, expectations and goals, attention, relation with pairs, direct observation of the real world, etc.

It is a question, however, whether merely providing information system support even having in mind improving situation awareness, is a sufficient solution. Many personal, organizational, and political factors influence acceptance of such systems, and in the context of functionally independent agencies there may be insufficient motivation to overcome these flaws. From a political point of view distrust might come from the fear to lose independence and autonomy whilst from an organizational point of view (i.e., within a given agency) systems might be rejected for the extra workload they imply.

This paper focuses on the acceptance process of a specific EMIS for inter-agency cooperation, SIGAME (Montells, Montero, Díaz, Aedo, \& de Castro, 2006) that has been developed for the Spanish Civil Protection Mechanism. Section 2 describes the system that was designed to meet known needs and mitigate know flaws. However, an empirical study reported in Section 3 demonstrates that end-users have continued to rely on traditional media, namely phone and fax. We use this empirical study to analyze in Section 4 some strategies to facilitate the acceptance of an EMIS in a multi-organizational context: applying a participatory design process to engage end-users as done before for other EMISs (Frishberg, 2005; Kristensen, Kyng, \& Palen, 2006) by integrating advanced tools providing users with experiences such as focused immersion, enjoyment, control, and curiosity that can improve cognitive absorption, and in turn acceptance (Agarwal \& Karahanna, 2000); and, finally, using the EMIS to build a community of practice crossing the boundaries of a single organization where interpersonal relationships among end-users of the involved agencies might help to generate a better response capacity (Waugh \& Streib, 2006).

\section{SIGAME in inter-agency cooperation}

SIGAME was designed to improve the co-ordination of inter-agency donations in emergency situations within the context of the Spanish Civil Protection Mechanism, which is made up of 19 autonomous agencies, one per each Autonomous Community or City, and a central government agency that only takes part in the emergency when requested by the local agency.

The system is an information and communication network (see Fig. 1) used both to collaborate in emergency situations and to communicate for other more general purposes in non-emergency situations; this dual usage reflects the basic principle that systems that are not used on a regular basis will not be used when the emergency occurs (Dykstra, 2003; Turoff, Chumer, Van de Walle, \& Yao, 2004). In fact, in (Landgren \& Nulden, 2007), authors show in a study involving two different incidents that first responders use the mobile phone in large-scale emergencies because this is their basic tool on normal conditions. Making the EMIS a tool that can be used not only in case of high-scale emergencies will contribute to make the system more likely to be used when the real crisis happen because the cost for adoption would have been gradually lowered. Both usages are implemented as clearly differentiated information flows and, consequently, they correspond to different parts of the system so that users are always aware whether they are dealing with an emergency (because they are in the area where information on emergencies is presented) or just using the system to communicate with their colleagues (because they are in the communication module). The central government also plays the role of mediator in international co-operation processes that can use SIGAME to coordinate the global Spanish response or to broadcast requests through international platforms (e.g., ARCE (Aedo et al., 2002) for the Latin American community or CECIS (EC, 2008) for the European Union). 


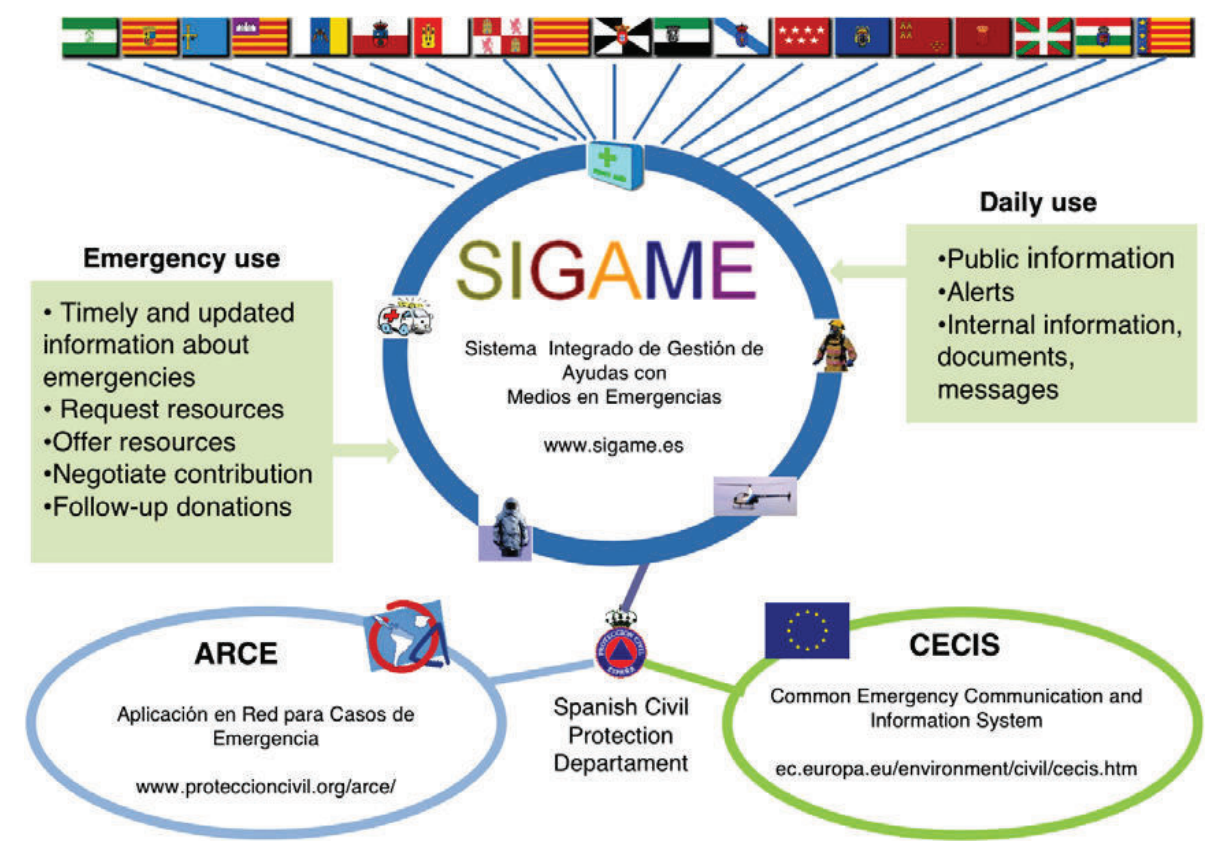

Fig. 1. SIGAME and its relations with other EMIS.

Reports from real cases of emergency response document flaws or challenges in cooperation among functionally independent governmental agencies (García-González, 2007; US House, 2006; Waugh \& Streib, 2006). Below we present these challenges and how they were addressed in SIGAME.

- Poor information sharing. Information overload, inconsistency, distrust or dearth, complicate the decision process that has to deal very often with incomplete or ambiguous data. For example, the massive fires of 2006 in Galicia (Spain) were managed by the regional governmental agency and required the cooperation of other 25 government agencies (17 regional, 3 national and 5 international). Requests were first asked by phone and then confirmed by fax. Reports on the response process show that information sent by fax didn't match always the phone request, giving place to a back-and-forth clarification process to determine accurately which resources were really needed. Indeed, one of the main conclusions of the committee analyzing the response to this emergency was "the need for mechanisms to broadcast updated information using clear and efficient communication channels that do not rely on individual efforts as it happens with traditional methods" (García-González, 2007). Such example, which is not unique, demonstrates that the lack of reliable information affects negatively to the situation assessments and ends up in an inefficient response.

To deal with these problems, SIGAME offers a common database about the emergencies, their situation, the resources required and the contributions already done or being negotiated by other agencies, so that each agency can have as much information as possible for improving their sense making process. The interface to the information base has been carefully designed in cooperation with real operators to try to make the system an aid in developing situation awareness. We cannot guarantee that decisions are the most rational ones, since coming out with a correct situation awareness is a personal process in the operators mind which is influenced by many factors and even studies demonstrate that a good situation awareness do not necessarily derive on good decisions (Endsley, 2000), but at least we want or EMIS not being a barrier to understand the situation and its implications. Experts had previous experience on this kind of situations as well as on the tools that operators use on a daily basis. Indeed they were very concerned about the information that had to be provided for each event in the system, to be sure that operators receive the information they need, in the format they understand and conveniently contextualized so that it can be understood. Thus, we could define for each kind of situation (such as alerts, requests of resources, offers or negotiations) all the items that were strictly necessary to understand not only the emergency and its consequences but also how other agencies are reacting to have a whole picture of the situation. Each information piece is accompanied by the identification of the user who generated it and the creation time, so that users are also aware of timeliness and authority as suggested in Turoff et al. (2004).

Moreover, experts were also aware that our EMIS is just one of the many tools operators use each day, so we couldn't expect users being continuously connected to SIGAME to realize when an event occurs. For that reason we decided to include an alert system that will send an email or an sms message to the operators whenever an important event that requires their attention occurs in SIGAME. Since these messages might be received outside the scope of SIGAME while operators are performing other tasks there is a need to avoid a poor contextualization of information that could result on poor situational awareness. For that reason messages are basically requests to access SIGAME were information is presented within its context as shown in Figs. 2a and 2b. 


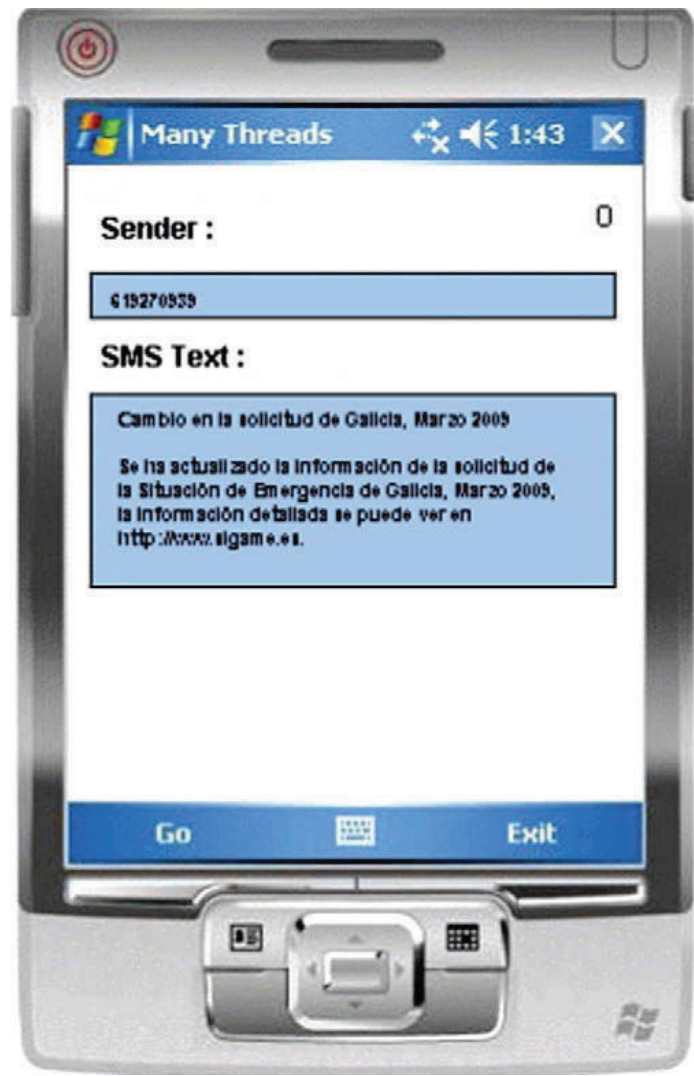

Fig. 2a. Example of message received in a mobile phone that informs that the status of Galicia emergency has changed and ask the operator to connect SIGAME to see the modifications

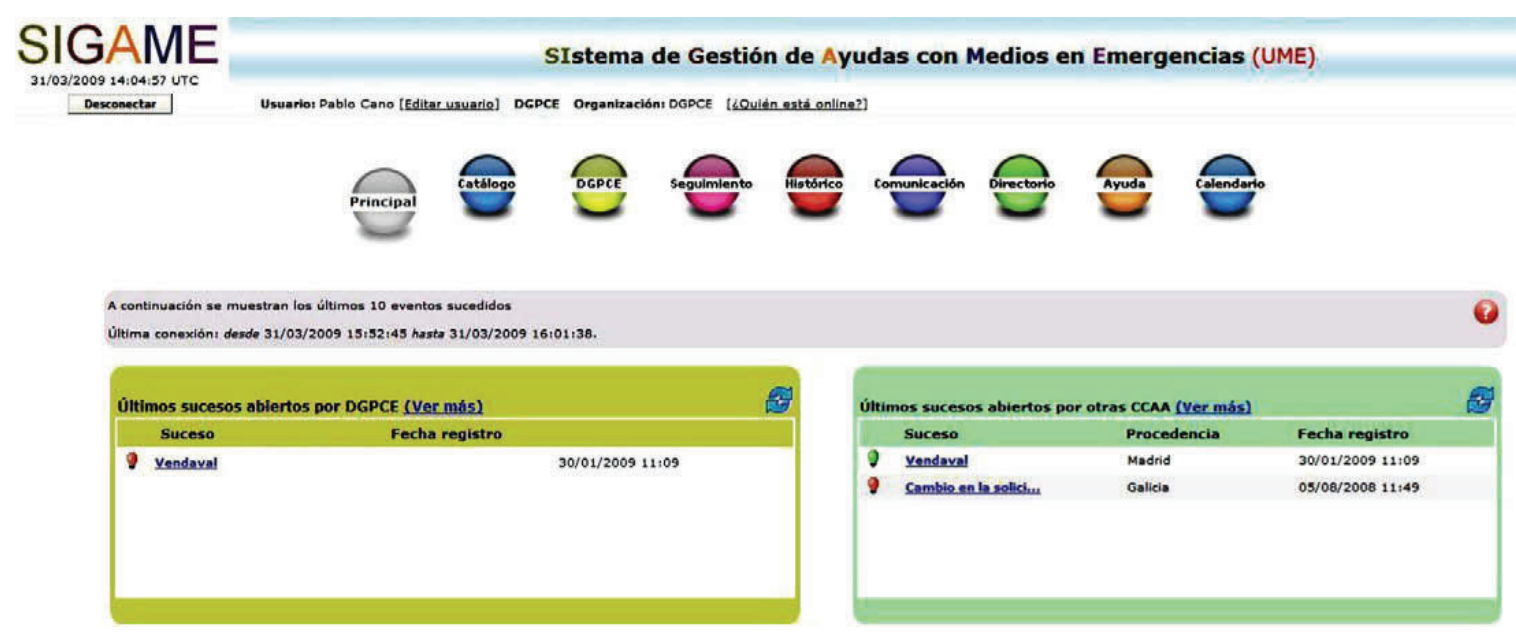

Fig. 2b. Entry panel of SIGAME where the last events are summarized. In the right pane, the second line (with the red icon before the item) is the link to the event for which the message in 2.a was sent. (For interpretation of the references to colour in this figure legend, the reader is referred to the web version of this article.)

- Lack of a fluent communication process. Whether because communication infrastructures are damaged and there is no way to establish a reliable connection or whether because responders do not know the right person they have to contact to, communication flaws provoke serious delays in the response. For example, during Katrina's response there was a huge communication problem due to the absence of infrastructure, namely satellite phones, in many parishes. This problem could have been quickly solved by FEMA, which owns mobile communication units that were not displaced to the affected area because none asked FEMA to do it, probably because none knew how to do it (US House, 2006). Physical communication has to be faced with adequate, updated and known communication protocols (Klenk, 1997), however even having a way to connect it still remains the problem of knowing who is the right person to be contacted. 
Communication in SIGAME follows an information flow policy where each user is notified of events updated in the system according to her role (Aedo, Díaz, \& Sanz, 2007). Users only have to add events to the system and decide if they want to use additional notification mechanisms (e.g., Fax and SMS). SIGAME uses its directory to send the notification to the right users taking into account the kind of event generated and the role of the user in the system. As a result, users need not to be aware of identifying the right person to send the information.

- Lack of coordination amongst agencies. Different agencies have different and sometimes overlapping protocols and not always share a unit of command. This makes it very difficult - if not impossible - to set up an efficient command-andcontrol structure. For example, reports on the Katrina response show lack of coordination not only at the inter-agency level but also at the intra-agency level (Waugh \& Streib, 2006).

There is a common protocol embedded into the SIGAME workflow. Simulations and drills are performed on a regular basis to build capacity on emergency response based on this protocol. Moreover, each agency has updated information on the situation as well as on the contribution of other agencies. In this way, we can support some level of activity awareness that might help agencies to make more informed and efficient decisions on how to cooperate in a specific emergency.

In a number of simulations and drills, domain experts had considered the SIGAME system useful for inter-agency cooperation. In these drills, operators and emergency managers of the different autonomous agencies are invited to run a simulation of emergency using the system and then provide impressions and opinions to improve it. In addition, we performed an empirical study to test whether the system was likely to be used in real emergencies.

\section{An empirical study on the use of IT for inter-agency cooperation}

The main motivation of the empirical study described in this section was to analyze how emergency workers of functional independent emergency management (EM) agencies collaborate among themselves and what is the role of IT in this process to try and envision potential strategies to improve the acceptance of a system like SIGAME. We also analyzed the familiarity of emergency workers with different technologies, to try to determine whether the reluctance to use an EMIS can be due to a personal attitude against technology. We conducted the study using questionnaires and follow-up interviews among SIGAME stakeholders. These included workers and managers of EM agencies of Autonomous Communities and Cities and representatives from the central government. In this section we briefly describe the study and the main findings.

\subsection{Information gathering}

This process was organized in three phases all of which are based on a common questionnaire.

In the first phase, a pilot test of the questionnaire was done to check its comprehensibility and utility. Thus, the questionnaire was first evaluated by five researchers in emergency management and then by three experts in civil protection. In the final questionnaire participants were asked about the use of technology in different ways, including a final section where they have to put such usage into the context of a real emergency situation they have been involved in.

During the survey administration, 32 emergency workers and managers belonging to different governmental agencies were asked to fill out a questionnaire that was organized into five parts:

1. Experience with tools: it gathered information about the frequency of use of technological tools (like web browsing and electronic mail) for work and for other purposes with a view to have an idea of the familiarity with IT tools of the respondents.

2. Experience in emergency management: it included questions to know the respondents position and expertise (both in terms of domain of expertise and duration of their experience).

3. Functions and tasks performed: it included questions about the functions and tasks they usually performed during the management of an emergency to be able to discriminate the pertinence of their answers for our purposes.

4. Working place, devices and tools: it was devoted to the description of the working place and collaboration with other people with a view to envision potential physical or organizational problems in the adoption of SIGAME.

5. Last Participations in resource management: it collects information like closure criteria, type of communication and used devices contextualized in the most recent participation of respondents in the resources management. It was used to check the validity of their comments.

In this paper, we will focus only on those questions strictly related with the use of technology, whether to collaborate or not. The questionnaire included also other parts intended to improving the Role Based Access Control policies applied in SIGAME (Aedo et al., 2007) so they go beyond the scope of this paper.

Responses were anonymous and evaluators were reminded that their information would not be disclosed to third parties. The survey respondents could not be paid, as they were all working for the government. We received 11 responses. Even though the questionnaire was anonymous, some participants included their position in the organization, which allowed us to determine which agency they were working for. Overall, we collected answers from 6 or more different organizations, across differ- 
ent levels within the organizations, and from organizations that were different for their size and/or available. Finally, three additional interviews were carried out with end-users using the same questionnaire so that we could delve more deeply into some questions and clarify some issues. Thus, even though the response set is relatively small (14 respondents, that is a $43.75 \%$ of the potential respondents) it includes a representative sample of relevant work positions and organizations with different sizes and availability of resources. This suggests that the measures collected have good validity with respect to the actual work practices. All the data combined with the experience reported from real situations in official studies like (García-González, 2007; US House, 2006) make it possible to derive some potential findings about the use of IT in emergency situations.

\subsection{Evaluation results and conclusions}

Results from questionnaires and interviews were analyzed and compared to understand how technology is being used in the cooperation among agencies, what are the user needs as well as the strategies that can promote a higher usage rate of EMIS for collaboration in real working settings.

The first aspect we analyzed was the users' experience with IT tools. Our results show that the EM workers use Internet, interactive maps and GPS for work purposes but, at the same time, they were familiar with using e-mail and audio conference to communicate for purposes other than work.

Concerning in what ways communication occurs during the collaboration process, a first result pertains to the kind of technology that the agencies used (i.e., traditional devices, that is phone and fax, vs electronic devices, like Internet, satellite phones and PDAs) and whether this usage depended or not on the number of interlocutors (see Fig. 3). Results show that multi-directional (one-to-many or many-to-many) communications were mainly done face to face and via traditional devices; one-to-one communications occurred via traditional devices. The use of electronic devices was in any case rare and limited to sending e-mails to multiple users.

When negotiating, emergency workers need devices to communicate and organize resources. In our study the participants preferred traditional devices to electronic ones. We classified users based on their work position to study correlations between tasks and technology usage. Comparing each classification with these results, we observed that the work position of the user does not influence significantly the frequency of use of different kinds of tools. We also classified EM workers according to their experience in terms of number of years spent working in the EM area. We categorized the respondents in three groups based on their years of work experience: less than 5, between 5 and 20 years, and more than 20 years. The three groups are quite homogeneous as the mean standard deviation in their answers in an interval of values [1,5] is $0,07,0,91$ and 0,91, respectively. In this case, results (see Fig. 4) show that users with 0-5 years of experience were frequent users of all kinds of tools whereas those with more than 20 years of experience choose web browser and electronic mail. These results suggest that less experienced workers might be more likely to use new technologies whilst workers with more extensive experience are more comfortable with basic tools (with worker's age as a potentially related factor). Analyzing how tool experience changes as the worker position changes, we have observed the same situation: a high frequency of use for web browser and electronic mail and a very low frequency for videoconference and other kinds of collaborative tools.

Overall, a basic finding was that most information, communication, and coordination currently rely on basic tools: phone, fax, and eventually other media like e-mail. This conclusion is also supported by documented cases of real emergencies that involved multiple agencies. For example, in the Katrina's response, in order to manage evacuation to Texas the main mechanism used was the phone (and web sites to get phone numbers from directories) and in some cases the e-mail, provoking a crash in the mail of one of the managers (FEMA, 2008b). Another finding was that there was no technophobia or a negative attitude against the use of technology. Therefore, there are appropriate conditions for an EMIS like SIGAME to achieve a good level of acceptance provided that appropriate strategies are used to promote acceptance.

\section{The acceptance of EMIS in multi-agency contexts}

To better understand the ongoing acceptance (or lack of acceptance) for SIGAME, we draw on the literature on the Technology Acceptance Model (TAM). According to the original proposal (Davis, Bagozzi, \& Warshaw, 1989) two parameters that gather subjective impressions of end users about a specific IT, the Perceived Usefulness (PU) and the Perceived Ease of Use

\section{People: kind of communication}

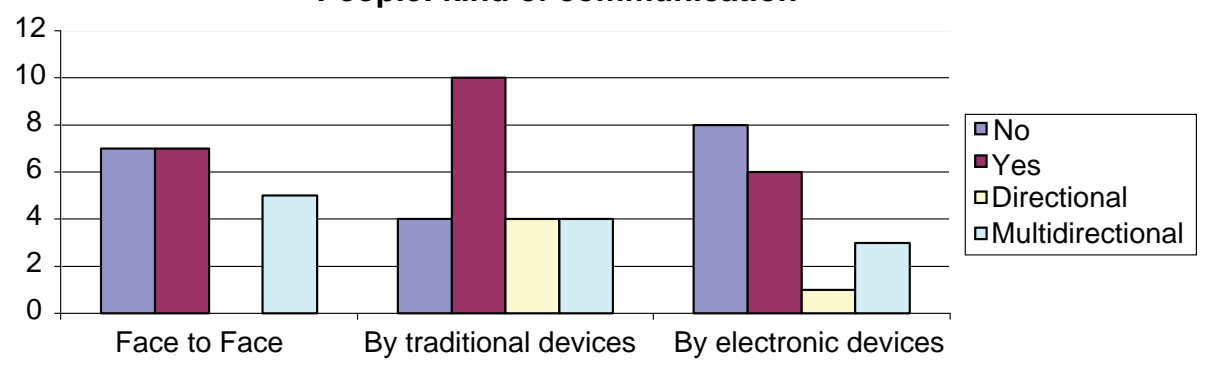

Fig. 3. Kinds of communication and tools usage. 


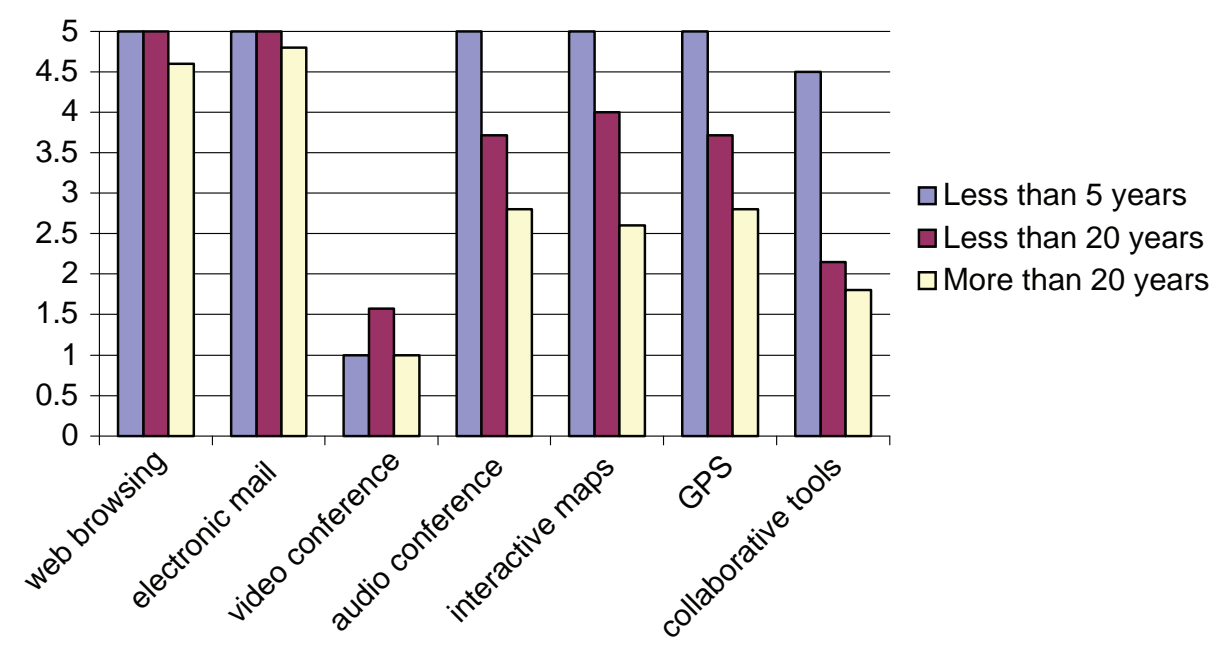

Fig. 4. Working experience vs. tool usage.

(PEOU), contribute to increase the Behavioral Intention of Use (BU), defined as "a measure of the strength of one's intention to accept an IT application". Furthermore, Chan and Teo show that PU has a stronger influence in BU than PEOU (Chan \& Teo, 2007), so as far as end users consider that "using a specific system will increase his or her job performance within an organizational context" (Davis et al., 1989), we could guarantee the system acceptance. In any case PU might not be enough in multiorganization settings as we have confirmed with SIGAME, whose users are quite satisfied with the system utility but they don't use it as our empirical study demonstrates.

As argued by Mathieson, Peacock, and Chin (2001), the TAM model assumes that "acceptance is volitional", that is it depends only on the end-users decision, but in an organizational context this is not the case. There are other parameters that influence the final adoption, including the Perceived Resources defined as "the extent to which an individual believes that he or she has the personal and organizational resources needed to use an IS" (Mathieson et al., 2001). To add more external variables to this model, in a multi-organizational setting, like the inter-agency cooperation process, each organization is autonomous and sovereign, and they can accept or not the system on an individual basis. This implies that we have to consider at least three different sources of external influences, not to mention legal constraints.

- Firstly, end users, who are the operators of the response centers, need not only be able to use a specific technology and be confident about its utility; they also need to trust it and be sure that its use does not imply any risk nor any loss of efficiency. Indeed, all the comments and suggestions we gathered from the SIGAME drills were always oriented towards making the system more efficient in terms of the relevance and utility of the information conveyed. Operators were also quite concerned about the extra burden that such a kind of system could imply but once they started using SIGAME they realized it was much more efficient than using traditional media like phone or fax to communicate with their pairs in other agencies.

- Secondly, emergency managers will be worried about losing autonomy, control and efficiency due to overlapping between the cooperation protocol and their own response protocol. However, we also observed that after the first drills this initial reluctance was somehow softened when managers realized that the system didn't interfere into their protocols.

- Finally, politicians will not accept any loss of autonomy on their decisions, not only for strategic reasons but also because in some cases it is a stronger requirement imposed by the legal nature of the involved agencies. In this case, it is never clear whether political support can or not be achieved. SIGAME has been designated by the Council of Ministries as the official tool for inter-agency communication in Spain but again, agencies are absolutely autonomous so they can decide on their own whether to use it or not. Whilst some of them consider SIGAME as a cheap way to improve their emergency management process, both in terms of funding (it has no cost for autonomous agencies) and in terms of political cost (this is a national initiative so responsibility can always be delegated), others might consider it as an imposition from the central government.

In order to improve the acceptance of SIGAME we are currently applying a number of strategies oriented towards winning acceptance by end users. Assuming that "emergency management capacity is built from the ground up" (Waugh \& Streib, 2006, p. 33), if end users are convinced that IT tools are useful for improving their performance they can raise some reaction at the political level and create a sort of bottom-up acceptance process. Moreover, it would be unwise for a specific agency to object to EMIS use if their workers and other peer agencies know that there it is a useful tool that will not interfere with their daily work nor lead to any loss of control. The strategies we propose include a participatory design process, the integration of advanced and enjoyable collaborative tools and the creation of a real community of end-users with a strong sense of contributing to do better a useful and personally rewarding work. 


\subsection{Participatory design in SIGAME}

SIGAME is being developed following a participatory design process organized in three different levels:

- Multidisciplinary development group. Software developers held a bi-weekly meeting with stakeholders, a group that includes end-users and a representative of the central government who also provides more than 25 years of fieldwork expertise in the management of international and national emergencies. As a research strategy alternative to ethnography (see studies by Kristensen et al. (2006) and Frishberg (2005)), we directly imported in the design process the experience of workers involved in real crisis management. Mock-ups and prototypes were used to elicit and specify the system requirements and features as in other participatory design projects. We also made use of visual notations of the software design using the ADM notation (Díaz, Montero, \& Aedo, 2005), which made non-technical designers to feel motivated. This kind of highly technical activity tends to nourish sense of personal innovativeness and ego satisfaction. In these meetings new ideas were discussed as well as the suggestions done by other end-users through the training program.

- Training program for end users. At least twice a year, there was a meeting or workshop with end-users of the different local emergency management agencies. A simulation of an emergency was used to assess the system and build emergency management capacity as suggested in Waugh and Streib (2006). In each drill, a different agency took the role of affected community and started the process by asking for resources. Moreover, surveys and open discussions were used to elicit ideas and requirements, whether concerning new services or the adaptation of the existing ones to the actual protocols of each agency. These ideas were discussed further in the multidisciplinary meetings and the results were also shared with this community of end-users during the next workshop. As pointed out by Kristensen et al. (2006), we realized that these workshops caused innovative ideas to emerge in an incremental way. Whilst in the first workshop most participants were quite passive, as they got more experienced with the system functions as well as more confident both on the development team and on the utility of their comments, they started to take active part on the discussions and even become the main advocates of the system.

- Political meetings. Once a year, the SIGAME status is discussed in political meetings with political representatives of all the involved agencies. In fact, the system has already been approved by the Council of Ministries as the tool to be used for cooperation inter-agencies.

Participatory design provided us a means for gathering requirements and specifications of needs directly from end users so that prototypes are iteratively refined to better address the detected needs. But it also has other benefits. First, keeps the design process open to innovative ideas from the development team as well as from end users. As more courses were held, end users got more and more engaged with the system. After the last course in April 2008, some of them formally requested a new course in June in order to be better prepared for the summer fires campaign. The positive attitude of EM workers helps to propagate the use of technology in a bottom-up way and eventually mitigates some of the political fears. Second, and from the point of view of entire organizations or agencies, the participation of their workers in the design process is perceived as a better approach than imposing a new system on the workers. Moreover, the participation in courses is seen as an investment for training the personnel. In fact, courses involve simulations that help to improve the response capacity of the workers. Finally, and from the point of view of political representatives, local governments can use the courses to publicize their initiatives to invest in technology and an integrated system to better respond to emergencies and at the same time this does not impose a direct cost for them.

\subsection{Integration of communication and coordination tools}

The combination of multiple agencies, their roles and policies, and tools used (including the EMS) constitute a working socio-technical system. The acceptance of the EMIS in this system, at a point in time, can be viewed as a summative function of the global fit between the needs of the actors (operators, agency managers, and politicians) and the functions supported by the EMIS. However, we argue that the etiogenesis of technology acceptance starts with the adoption at the level of individual users; then, over time, this triggers adjustments at higher levels of the socio-technical system. Thus it is critical to promote adoption at the level of the individual emergency workers: meeting their actual needs, increasing their benefits, and reducing their costs.

About supporting the right needs, during the phase of emergency response across multiple agencies the basic functions are sharing knowledge about the emergency, coordinating response actions, and keeping in touch. The support for these functions needs to be efficient, reliable, and at low cost. New collaborative technologies include tools that successfully support these functions. Ellis, Gibbs, and Rein (1991) classify collaborative tools based on the main function they support: communication (i.e., exchange of messages), coordination (i.e., manage people, activities and resources), cooperation or coproduction (joint production in a shared space). In terms of this classification the tools that are useful for emergency response are primarily communication and coordination tools. In SIGAME the tools provided for everyday usage and during emergencies include the followings:

- Common information repositories and shared catalogues of resources are used to improve communication by disambiguating terms. In order to deal with local terminologies, each agency can add to each entry its own aliases. These information repositories have been accepted by all agencies. 
- The tools for managing the donation of resources support an open and multidirectional negotiation process. Agencies are always aware of the status of the negotiation process. The system provides timely and trustable information about the situation and the responses by other agencies. Information flows to all the agencies at the same time and even alternative media, including fax and SMS, can be used to notify relevant events.

- Notifications and cues of users presence in the system are two mechanisms that help users to maintain a realistic and upto-date picture of what is happening: who is in the system and how they are reacting.

- A multidirectional communication module makes it possible to send messages to the SIGAME users and to broadcast different kinds of news whether public (alerts, RSS and press releases) or not (general information about emergencies and civil protection and SIGAME related news).

But having tools that are useful (i.e., support the right function) does not ensure acceptance. A later version of the TAM theory broadened its analytical-cognitive perspective introducing the construct of cognitive absorption as important precursor of the users personal beliefs about utility and usability. Cognitive absorption is a state of deep involvement or a holistic experience of a user interacting with a given technology (Agarwal and Karahanna, 2000). Typical episodes of cognitive absorption appear when interacting with videogames and the Internet. This broader perspective is more compatible with emerging design approaches that focus on improving the holistic experience rather than maximizing specific user responses. In order to successfully promote acceptance, collaborative tools should not only be useful, usable, and easy to learn, but also should induce in the emergency workers experiences such as focused immersion, enjoyment, control, and curiosity (Carver and Turoff, 2007). In this way, they can engender some level of cognitive absorption, which will impact positively on the Behavioral Intention of Use (see Zhang, Li, \& Sun, 2006, on cognitive absorption of users of a website). For example, the tools that support awareness (e.g., peripheral cues about collaborators' presence, actions, and objects) and response coordination (e.g., task manager, checklist, members' roles and relationships) can increase the level of cognitive absorption. Indeed, as pointed out in (Carver \& Turoff, 2007) there are five characteristics of the concept of cognitive absorption that apply to emergency workers: (1) a complete focus on the task being performed; (2) a high motivation due to the critical nature of the system and the responsibility of the worker; (3) role players (emergency workers in our case) exercise control; (4) users have to improvise since they do not have all the information; and (5) there is a continuous sense of challenge and probably curiosity. One might argue that such a highly focused attention might be controversial, as operators cannot realize changes in their environment but SIGAME makes use of visual clues to attract the attention of operators whenever it is required to avoid operators losing focus on the situation.

If we can assume that the technology is useful, cost-effective and engaging and that there is group of early adopters, then collaborative technology may exhibit a specific advantage in spreading adoption, in comparison to individual technology. There are two reasons supporting this hypothesis: the influence of peers and the network effect. First, emergency workers typically collaborate with partners whom they know, trust, and respect. Research on innovation shows that people are more likely to adopt a technology if other people they know and respect have also adopted this technology. Second, the overall speed of technology adoption is determined by the speed at which adoption takes off and by the speed at which occurs later. Even if in the short term individual technology may spread at a higher initial speed, in the long term collaborative technology benefits incrementally from the network effect, as it happened for fax or email technologies; that is, as a critical mass of adopters is reached then the benefits of adopting the technology increases continuously as more people get it (Rogers, 2003).

\subsection{Building communities of practice in multi-organizational cooperation}

When multiple agencies (official and non-governmental) are involved in responding to an emergency, the immediate goal is to coordinate efficiently and respond effectively. The long-term goal is to maximize the resilience of the network involved for when emergencies strike again. In most countries, the approach to achieve these goals has been limited to top-down initiatives directed by governmental agencies (e.g., US Dept. of Homeland Security). These tend to focus on ensuring infrastructures (e.g., transportation, EMIS), division of labor among people (e.g., roles), and standardization of the behaviors (e.g., communication protocols, explicit plans). However, the above goals can be better achieved if the top-down activities are complemented with bottom-up community-based initiatives (Waugh \& Streib, 2006).

In emergency management, the community-based approach has only been used at the level of local collocated communities, where a few interdependent agencies operate within a geographical community, as in Schafer, Carroll, Haynes, and Abrams (2008), where researchers found that the emergency planning activities are inherently collaborative and are shaped by the geography, experts, resources, and culture of the community. However, what we are proposing here is a different kind of community, specifically, supporting the generation of communities of practice among emergency management workers from the different agencies. This kind of community of practice would help cumulating and transferring expertise and improve the capacity to respond to emergencies over time. For example, Landgren and Nulden (2007) investigated the response to two major chemical hazard incidents in Sweden and found that mobile phones enabled emergency responders across organizational boundaries to socially form and manage a network of work and thereby improving the capacity of enacted sense making. In a completely different domain like the academic community using Citeseer, Farooq, Ganoe, Carroll, Councill, and Giles (2008) suggest the role of the community of practice to enhance individual sense making through awareness of what collaborators are doing. This is a claim we have heard in all the drills and simulations of SIGAME and poses challenging requirements in the design of communication tools to promote the generation of effective communities of practice. 
Therefore, community-oriented EMISs could integrate a suite of tools providing connectivity and networking at low cost in different situations, such as Instant Messaging (or text messaging), Voice over IP, or videoconferencing to form the community of practice in normality situations, when collaborators from different agencies can share experiences about the problems and the solutions as well as informal sources of information that are so important in busy units like operation centers for medical emergency (Reddy \& Spence, 2008). In this way, IT technologies can help to share knowledge of resources, tested practices, and to create a sense of membership that fosters mutual support among the members and greater acceptance of the EMIS, as a community medium. In this sense, a parallel could be drawn with Orr's study (Orr, 1996) of how a community of practice of experienced technicians at Xerox shared knowledge. Similarly to emergency management workers, Orr's technicians were experts who regularly had to diagnose problems, find solutions, and reuse knowledge as they move from fixing one problem to the next. He found that their knowledge about the diagnosis and the process circulated through "war stories" or anecdotes among the technicians. This form of peer-to-peer knowledge coordination was more effective than the official manuals distributed (top-down) by the company and, in the long term, changed the dynamics of the company.

In our case, we intend to provide emergency management workers with tools to share their experience and expertise. We have observed in the drills that while at the beginning they asked to the development team members for advice they soon turned to lean on other participants and they also started to discuss other issues related to their work as they were eager to comment their own experiences and problems. This made them feel more comfortable with the drill and also contributed to reaffirm their knowledge on the management process or on the tool usage. In this way, if we are able to deploy tools that can be used to establish a continuous and informal communication flow between emergency workers we could contribute to create a real community of practice on emergency management and then rely on this community and its commitment with the tool as the real engine to get a full acceptance of the tool. Moreover, the interaction that takes place in a community of practice is a powerful means of social learning (Lave \& Wenger, 1991) so that if adequate tools are developed we can extend the scope of SIGAME to support informal preparedness of novice emergency workers.

\section{Conclusions}

The cooperation among autonomous governmental agencies of emergency response is not always an efficient process. Problems due to lack of information, disruption of communication, or poor coordination delay response and make it inefficient. Information systems could help to solve some of these problems, as far as they provide a way to share information and implement communication flows and coordination workflows. However, the main challenge when developing IS for interagency cooperation is to get a final acceptance from all the involved agencies.

Developers cannot just rely on the quality of the system and then expect users will use it. Some strategies have to be applied in order to push the acceptance process, for which we propose focus on end-users and rely upon a ground up process, where political and organizational managers accept the system because end-users are willing to use it. The strategies we proposed include a participatory design process that involves end-users in most design decisions, the inclusion of communication and coordination tools that could induce in the emergency workers cognitive absorption experiences and consequently increase the behavioral intention of using the system and, finally, the use of the system to create a community of practice.

\section{Acknowledgements}

This work has been funded by the Grant (PR2007-0271) and the Research Project (TSI2007-60388) of Spanish Ministry of Education and Science. SIGAME is a project funded by Dirección General de Protección Civil y Emergencias of Spanish Ministry of Interior. Authors would like to thank Susana Montero, David Diez and Laura Montells for their work in SIGAME and Helena Mentis, Jorge de Castro and Carmen Cobo for their comments to the interviews.

\section{References}

Aedo, I., Díaz, P., Fernández, C., \& de Castro, J. (2002). Supporting efficient multinational disaster response through a web based system. In E Gov conference. (pp. 215 222), Aix en Provence, Francia. September.

Aedo, I., Díaz, P., \& Sanz, D. (2007). A model based approach to specify the access needs of emergency management communities using RBAC principles. Special issue on emergency management systems. Journal of Intelligent Control and Systems, 11(4), 272283.

Agarwal, R., \& Karahanna, E. (2000). Time flies when you're having fun: Cognitive absorption and beliefs about information technology usage. MIS Quarterly, 24(4).

Carroll, J. M., Neale, D. C., Isenhour, P. L., Rosson, M. B., \& McCrickard, D. S. (2003). Notification and awareness: Synchronizing task oriente collaborative activity. International Journal of Human Computer Studies, 58, 605632.

Carver, L., \& Turoff, M. (2007). Human computer interaction: The human and computer as a team in emergency management information systems Communications of the ACM, 50(3), 3338.

Chan, H. C., \& Teo, H. (2007). Evaluating the boundary conditions of the technology acceptance model: An exploratory investigation. ACM Transaction on Computer Human Interaction, 14(2), 9.

Davis, Fred. D., Bagozzi, Richard. P., \& Warshaw, Paul. R. (1989). User acceptance of computer technology: A comparison of two theoretical models. Management Science, 35(8), 9821003.

Dykstra, E. H. (2003). Towards an international system model in emergency management. In Concept paper call for papers for the international conference towards an international system model in emergency management.

Díaz, P., Montero, S., \& Aedo, I. (2005). Modelling hypermedia and web applications: The Ariadne development method. Information Systems, 30 (8), 649 673.

Ellis, C. A., Gibbs, S. J., \& Rein, G. L. (1991). Groupware Some issues and experiences. Communications of the ACM, 34(1), 38 58. 
Endsley, M. R. (2000). Theoretical underpinnings of situation awareness: A critical review. In M. R. Endsley \& D. J. Garland (Eds.), Situation awareness analysis and measurement. Mahwah, NJ: LEA.

European Community. (2008). The common emergency communication and information system (CECIS). <http://ec.europa.eu/environment/civil/cecis.htm>. FEMA (2008a). National Integration Center Incident Resource Inventory System. <http://www.fema.gov/emergency/nims/rm/iris.shtm> Accessed 30.05.08. FEMA (2008b). Harris County, Texas Citizen Corps' Response to Hurricane Katrina. <http://www.fema.gov/pdf/emergency/nims/ lessons_learned_tx_katrina.pdf> Accessed 30.05.08.

Farooq, U., Ganoe, C. H., Carroll, J. M., Councill, I. G., \& Giles, C. L. (2008). Design and evaluation of awareness mechanisms in CiteSeer. Information Processing and Management, 44(2), 596612.

Frishberg, L. 2005. Looking back at plan AHEAD ${ }^{\mathrm{TM}}$ : Exercising user centered design in emergency management. In CHI '05 extended abstracts on human factors in computing systems, Portland, OR, USA, April 02 07, 2005. New York, NY: ACM. pp. 9881003.

García González, J. M. (2007). La coordinación de la emergencia desde la Administración General del Estado. Jornadas Técnicas: Coordinación administrativa de grandes incendios en la interfaz urbano forestal. <http://www.proteccioncivil.org/centrodoc/jt_cagincendios_2007/Ponencias/P2_PON9.pdf> Accessed 30.05.08.

Klenk, J. S. (1997). Emergency information management and telecommunications. Disaster Management Training Programme, DHA.

Kristensen, M., Kyng, M., \& Palen, L. (2006). Participatory design in emergency medical service: designing for future practice. In R. Grinter, T. Rodden, P. Aoki, E. Cutrell, R. Jeffries, \& G. Olson (Eds.), Proceedings of the SIGCHI conference on human factors in computing systems, Montréal, Québec, Canada, April 22 27, 2006, CHI '06. (pp. 161 170), New York, NY: ACM.

Landgren, J. \& Nulden, U. (2007). A study of emergency response work: patterns of mobile phone interaction. In Proceedings of the SIGCHI conference on human factors in computing systems, San Jose, California, USA, April 28 May 03, 2007, CHI '07. (pp. 1323 1332), New York, NY: ACM.

Lave, J., \& Wenger, R. (1991). Situated learning. Laegitimate peripheral participation. Cambridge: University of Cambridge Press.

Malone, T. (2001). In T. Malone, L. \& Erlbaum (Eds,). Coordination theory and collaboration technology.

Mathieson, K., Peacock, E., \& Chin, W. W. (2001). Extending the technology acceptance model: The influence of perceived user resources. SIGMIS Database, 32(3), 86112.

Montells, L., Montero, S., Díaz, P., Aedo, I., \& de Castro J. (2006). SIGAME: Web based system for resources management on Emergencies. ISCRAM 2006, Newark (USA).

Orr, J. E. (1996). Talking about machines: An ethnography of a modern job. New York: Ithaca.

Reddy, M. C., \& Spence, P. R. (2008). Collaborative information seeking: A field study of a multidisciplinary patient care team. Information Processing $\mathcal{E}$ Management, 44(1), 242255.

Rogers, Everett. M. (2003). Diffusion of innovations (5th ed.). New York, NY: Free Press.

Schafer, W. A., Carroll, J. M., Haynes, S. R., \& Abrams, S. (2008). Emergency management planning as collaborative community work. Journal of Homeland Security and Emergency Management, 5(1)

Turoff, M., Chumer, M., Van de Walle, B., \& Yao, X. (2004). The design of emergency response management information systems (ermis). Journal of Information Technology Theory and Applications, 5(4), 136.

US House Select Bipartisan Committee to Investigate the Preparation for and Response to Hurricane Katrina (2006). A Failure of Initiative. Washington, DC: US Government Printing Office. <http://katrina.house.gov/full_katrina_report.hm> Accessed 30.05.08.

Waugh, W. L., \& Streib, G. (2006). Collaboration and leadership for effective emergency management. Public Administration Review, 66(s1), 131 140. doi: $10.1111 /$ j.1540 6210.2006.00673.x.

Zhang, P., Li, N., \& Sun, H. (2006) Affective quality and cognitive absorption: Extending technology acceptance research, In Hawaii international conference on system sciences (HICSS), January 2006. 